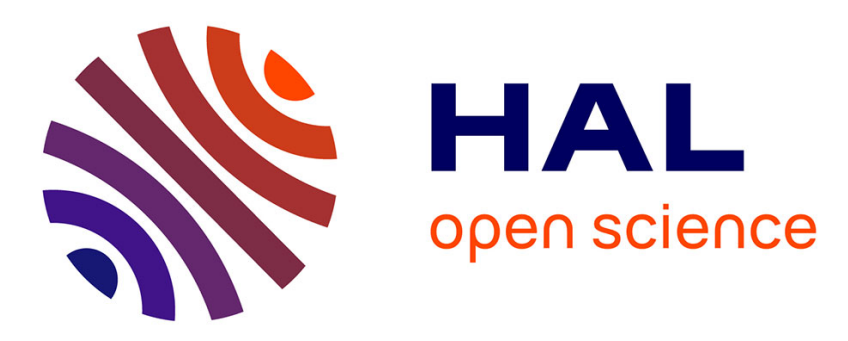

\title{
Quatre manières de contribuer à la satisfaction du patient hôspitalisé: Les éléments Basiques, Plus, Clés et Secondaires.
}

Sylvie Llosa

\section{- To cite this version:}

Sylvie Llosa. Quatre manières de contribuer à la satisfaction du patient hôspitalisé: Les éléments Basiques, Plus, Clés et Secondaires. . Journal de gestion et d'économie médicales, 2012, 30 (6). hal-01793171

\section{HAL Id: hal-01793171 \\ https://hal.science/hal-01793171}

Submitted on 16 May 2018

HAL is a multi-disciplinary open access archive for the deposit and dissemination of scientific research documents, whether they are published or not. The documents may come from teaching and research institutions in France or abroad, or from public or private research centers.
L'archive ouverte pluridisciplinaire HAL, est destinée au dépôt et à la diffusion de documents scientifiques de niveau recherche, publiés ou non, émanant des établissements d'enseignement et de recherche français ou étrangers, des laboratoires publics ou privés. 
Quatre manières de contribuer à la satisfaction du patient hospitalisé :

Les éléments Basiques, Plus, Clés et Secondaires

Four ways to contribute to patient satisfaction in hospital:

Basic, Plus, Key and Secondary elements

Sylvie Llosa

Professeur - IAE Aix en Provence - Aix Marseille Université

Centre d'Etudes et de Recherche en Gestion d'Aix - Marseille, CERGAM, EA 4225

IAE Aix en Provence

Clos Guiot Puyricard- CS 30063 - 13089 Aix en Provence Cedex 2

Tel : 04.42.28.08.33 / Fax : 04.42.28.08.00

sylvie.1losa@iae-aix.com

Article publié en decembre 2012 dans Journal de gestion et d'économies médicales (nouveau nom du Journal d'Economie Médicale)

vol $30 n^{\circ} 6,367-387$. 


\section{Quatre manières de contribuer à la satisfaction du patient hospitalisé : Les éléments Basiques, Plus, Clés et Secondaires}

\section{Résumé :}

L'objectif de cette étude est de mieux comprendre la construction de la satisfaction des patients hospitalisés en cliniques privées. Elle souhaite constater et vérifier qu'il existe quatre modes de contribution des éléments d'un séjour hospitalier à la satisfaction des patients. Elle se réfère au modèle Tétraclasse de Llosa [1] utilisé largement dans d'autres catégories de services. A côté de l'attention et de l'information donnée au malade et à ses proches qui sont déterminants et prioritaires, l'étude souligne le rôle particulier des critères hôteliers et notamment de la restauration comme élément de différentiation. Cette analyse pragmatique permet aux dirigeants d'établissement de mieux exploiter l'information contenue dans les enquêtes de satisfaction et de hiérarchiser les actions à entreprendre pour améliorer la satisfaction.

\section{Mots clés :}

Satisfaction, santé, cliniques, restauration, tétraclasse, contributions asymétriques.

The objective of this study is to better understand the construction of satisfaction of hospitalized patients in private clinics. It wants to show and verify that elements of a hospital stay contribute to overall patient satisfaction following four ways. It is based on the Tetraclass model from Llosa [1] widely used in other types of services. Along with the attention and the information given to the patient and his family which stay determinant and a priority, the study highlights the special role played by hotel criteria and particularly catering as a differentiating element. This pragmatic analysis enables leaders to better use the information contained in satisfaction surveys and to prioritize actions to improve satisfaction.

\section{Key words :}

Satisfaction, health, clinics, catering, tetraclass, asymetrical weights. 


\section{Quatre manières de contribuer à la satisfaction du patient hospitalisé : Les éléments Basiques, Plus, Clés et Secondaires}

Thème important de recherche, considérée par les praticiens comme une condition préalable à l'intention de réachat, à la fidélisation et à un bouche-à-oreille favorable [2,3], la satisfaction des clients est devenue une préoccupation majeure pour les entreprises privées. De façon plus récente elle intéresse également les établissements de santé [4] dans le cadre de la certification obligatoire des établissements, remise en cause tous les quatre ans. L'environnement se caractérise par une concurrence accrue entre établissements, une évolution du mode de financement de ces derniers (T2A). Si historiquement, les praticiens étaient les principaux vecteurs d'attraction vers les cliniques et hôpitaux privés, ces établissements souhaitent aujourd'hui établir une relation plus directe avec leurs patients. Fidéliser la patientèle, en particulier sur les maladies chroniques, devient déterminant. Des dispositifs d'écoute clients se généralisent avec par exemple la création de questionnaires de satisfaction communs (22 CRHU se sont regroupés dès 2007 pour une enquête coordonnée).

La satisfaction globale d'un patient hospitalisé est liée à l'évaluation des soins et gestes qui lui sont prodigués, aux informations et à l'écoute qui lui sont données ainsi qu'à son entourage, à la fluidité du processus, aux compétences techniques et relationnelles du personnel, etc. En parallèle de l'aspect purement médical, on parle aujourd'hui d'hôtellerie de santé pour qualifier toutes les attentions aux patients qui ne sont pas directement du soin. Certaines relèvent du confort matériel du patient (confort et propreté de la chambre, repas) d'autres sont de nature administratives (service d'admission, accueil principal de l'établissement). La qualité perçue de ces critères est souvent mesurée grâce à des questionnaires. Cependant au-delà d'une note de performance (souvent élevée), il faut comprendre l'importance relative de ces critères sur la satisfaction globale pour agir et arbitrer entre actions prioritaires, sous contrainte de budget. Ainsi, comment les patients combinent-ils les évaluations d'éléments individuels de service pour former un jugement global de satisfaction?

Notre recherche se focalise sur ces préoccupations. Elle souhaite constater et vérifier que tous les éléments d'une expérience de service ne contribuent pas à la satisfaction globale du patient en suivant la même logique. Pour cela, le modèle Tétraclasse de Llosa [1], jusqu'à présent utilisé dans d'autres 
catégories de services, est mobilisé et appliqué au secteur de la santé. L'étude se déroule dans les services chirurgie d'un acteur européen gérant des cliniques privées. Ce travail de réplication, catégorie de recherche dont l'importance a encore été récemment démontrée [5], permet de comprendre le rôle des différentes caractéristiques d'un séjour d'un patient hospitalisé dans sa satisfaction. Ce modèle fait l'hypothèse qu'il existe deux logiques de contribution principales à la satisfaction, chacune se divisant en deux modes secondaires :

- $\quad$ Le poids de certains éléments dans la satisfaction globale du client est dynamique, fluctuant. Il dépend du niveau de performance perçu par le client de ces éléments. Cette dépendance peut être soit positive (éléments Plus), soit négative (éléments Basiques).

- D'autres éléments contribuent à la satisfaction du client de façon stable, quelle que soit l'évaluation qui en est faite. Cette contribution stable peut être soit forte (éléments Clés), soit faible (élements Secondaires).

Dans cette optique, nous présentons d'abord le cadre conceptuel de la recherche, justifions le choix et l'intérêt du modèle Tétraclasse, le présentons de façon détaillée ainsi que les hypothèses qui en découlent. Ensuite, la méthodologie de cette étude et analysée. Elle est suivie de la présentation des résultats. Une discussion s'ouvre alors sur les implications managériales de l'étude. L'article conclut sur les apports et limites de cette dernière, et présente des voies de recherche futures.

\section{CADRE CONCEPTUEL, MODELE ET HYPOTHESE}

La satisfaction peut être définie comme «un état psychologique provenant d'un processus d'évaluation affectif et cognitif qui survient lors d'une transaction spécifique » [6].

Il existe, dans la littérature, deux approches principales de la satisfaction :

- la première analyse les processus qui engendrent la satisfaction, la mécanique sous jacente. Le paradigme de la disconfirmation ${ }^{1}$ (la satisfaction nait quand le service perçu par le client est supérieur

\footnotetext{
${ }^{1}$ Pour un état de l'art, voir [7].
} 
ou égal à ses attentes, l'insatisfaction se produit dans le cas contraire), ou d'autres théories, comme celles de l'équité ${ }^{2}[8]$.

- la seconde, dans laquelle s'inscrit cette recherche, s'intéresse aux modes de contribution des attributs à la satisfaction globale. Quantités de méthodes coexistent, certaines envisageant le poids des attributs de manière linéaire (modèles de décomposition ou de composition ${ }^{3}$ ), d'autres, plus récentes, de façon fluctuante.

Pour la majorité des chercheurs, la satisfaction est conceptualisée comme un continuum unidimensionnel opposant deux pôles extrêmes : positif (très satisfait) et négatif (très insatisfait) [10,11]. D'autres auteurs en marketing, influencés par les travaux d'Herzberg et al. [12] considèrent que la satisfaction est bi-dimensionnelle $[13,14,15]$ : les facteurs qui engendrent la satisfaction sont différents et indépendants de ceux qui engendrent l'insatisfaction ${ }^{4}$. Une troisième catégorie de chercheurs réconcilie ces deux visions : la satisfaction est unidimensionnelle, mais la fonction de contribution de certains éléments à la satisfaction est non linéaire ou encore asymétrique. Les méthodes de simulations de Kano et al. [16], de "pénalité/récompense" de Brandt [17], le modèle tétraclasse de Llosa [1], de Mittal et al [15] vont dans ce sens. Le modèle tétraclasse possède en particulier une validité externe solide puisque l'existence de ces quatre types d'éléments n'a pas été infirmé dans diverses recherches fondées sur des activités de services très variées (Banque, Restauration [1] ; Grande Distribution [18] ; Zones commerciales [19] ; Service Chirurgie court séjour [20]; Assurances [21]; Hôtellerie [22]. De plus, la fiabilité de ce modèle est confirmée par les travaux de Ray et Gotteland [23]. Cependant, la méthodologie développée par l'auteur n'est pas sans limite notamment en matière de validité convergente et se positionne clairement comme une approche exploratoire. Les avantages de ce modèle sont les suivantes :

2 L'idée est d'inclure ici une notion d'équilibre : le client évalue le service reçu par rapport à son coût (temps, effort, participation, argent). Puis, il compare son ratio "ressources engagées / résultats" avec celui de la personne de référence, celle-ci étant, dans la littérature marketing le vendeur, le personnel en contact ou l'entreprise qui délivre le service. La satisfaction client naît du degré d'équité perçue entre ces deux ratios ou encore lorsque le ratio client est supérieur ou égal à celui de l'entreprise.

${ }^{3}$ Pour une synthèse, voir [9].

${ }^{4}$ La plupart d'entre eux ajoutent aux facteurs de satisfaction et aux facteurs d'insatisfaction d'autres catégories d'éléments : des facteurs "hybrides" jouant fortement sur la satisfaction et l'insatisfaction ou des facteurs "neutres" ayant toujours un faible impact, quelle que soit leur évaluation par le client. 
- Il a été conçu pour analyser la satisfaction vis-à-vis d'une expérience de service (et non vis-à-vis d'un produit),

- Outre le fait de révéler plusieurs logiques de contribution, ce modèle ${ }^{5}$ a l'intérêt de déduire les poids des différents éléments de service de calculs statistiques (signe de mesure valide) et ne demande pas au répondant de «déclarer» l'importance des éléments ce qui alourdit considérablement un questionnaire. Il peut donc être réalisé sur la plupart des enquêtes de satisfaction déjà existante (questionnaire HAS par exemple), pour peu que la liste des éléments de service soit pertinente.

- la contribution des éléments est mesurée à partir d'évaluation d'expériences de service réellement vécues (et non simulée). Or, la satisfaction est, par nature, une évaluation post achat, fondée sur une expérience réelle,

- il demande uniquement des données ordinales, qui correspondent à la nature réelle de nombreuses échelles de satisfaction à support sémantique.

- De plus, il aboutit à des cartes visuelles, facile à comprendre et à communiquer.

Le Modèle Tétraclasse propose deux modes principaux de contribution, chacun se divisant en deux modes secondaires (Cf. figure 1) :

\section{Insérer Figure 1}

Ce modèle comporte deux axes, l'abscisse représente la contribution des éléments au niveau de satisfaction lorsqu'ils sont perçus de façon négative par les clients, l'ordonnée leurs contributions lorsqu'ils sont perçus de façon positive.

Dans le premier mode de contribution, le poids de certains éléments dans la satisfaction globale du client est stable, quelle que soit l'évaluation qui en est faite. Cette contribution stable peut être toujours forte, ce sont les éléments «Clés» ou toujours faible, les éléments «Secondaires ». Les éléments «Clés » sont attendus et déterminants. Leurs performance est éminemment sensible dans l'esprit des individus. Ils constituent souvent la raison d'être du service. Les éléments «secondaires » sont

\footnotetext{
${ }^{5}$ Pour une analyse critique et une comparaison des différentes méthodologies existantes voir [21, 23]
} 
importants car tout compte dans une activité de service, mais sont relativement moins déterminants dans la satisfaction du client.

Dans le second mode de contribution, les éléments contribuent à la satisfaction du client de façon fluctuante. Le poids des éléments dépend de leur niveau de performance perçu par le client. On distingue alors les éléments «Basiques » et les éléments «Plus »: lorsqu'un élément «Basique » est évalué négativement par le client, il influence fortement son niveau de satisfaction globale ; une évaluation favorable, par contre, a peu d'impact sur sa satisfaction, elle est attendue, implicite, c'est la moindre des choses dans l'esprit du client. Les éléments «Plus» ont une logique de contribution inverse, s'ils sont bien perçus ils augmentent le niveau de satisfaction globale, sinon ils ont peu d'influence. Une mauvaise performance ou une absence de ces éléments correspondant aux attentes prévues du client, il s'agit souvent de surprise, de gestes et d'attentions imprévus.

La réplication d'un tel modèle dans le secteur des structures hospitalières nous parait d'autant plus intéressante que ce dernier mesure la satisfaction de ses patients de façon relativement récente et que le sujet est éminemment impliquant pour tout individu. Les critères pris en compte par les patients pour évaluer leur satisfaction commencent à être connu de façon plus ou moins fine, mais la mesure de leur poids relatif dans la construction de la satisfaction demeure inexistante ou, en tout cas, perfectible. A notre connaissance seule Merdinger- Rumpler [20], en s'appuyant sur le modèle tétraclasse de Llosa [1] aborde pour la première fois dans le domaine de la santé humaine ce sujet, dans un service chirurgical public de court séjour.

L'objectif est ici de vérifier l'existence d'éléments « Basiques », «Plus », « Clés » et « Secondaires » dans le cadre d'un hôpital privé et d'apporter ainsi une meilleure compréhension à la construction de la satisfaction du patient. Nous proposons donc l'hypothèse classique suivante :

H1 : Dans un service de chirurgie d'un hôpital privé, nous retrouvons les quatre catégories d'éléments du modèle Tétraclasse de Llosa (1997), les éléments "Basiques", "Plus", "Clés" et "Secondaires".

Par ailleurs, Merdinger- Rumpler [20] a montré dans sa recherche sur l'hôpital public de court séjour que les éléments relevant de l'hôtellerie de santé (confort de la chambre, lits, sanitaires, calme) étaient 
des éléments «Secondaires». Seul les repas à l'hôpital étaient un élément « Plus », à la frontière des éléments «Secondaires ». Nous supposons que dans le cadre d'une clinique privé et avec des durées de séjour plus longues, la restauration, comme la plupart des éléments hôteliers liés au confort, joueront plus fortement sur la satisfaction du patient en cas de bonnes prestations, gardant un poids faible si le niveau de prestation laisse à désirer. Nous émettons donc l'hypothèse suivante :

H2 : Les éléments liés au confort hôtelier (confort de la chambre, restauration, calme, propreté) sont des éléments «Plus ».

\section{METHODOLOGIE}

L'identification des éléments qui contribuent à la satisfaction est d'abord nécessaire. Le réseau de cliniques privées étudié avait déjà un questionnaire de satisfaction en place. Malgré les limites de ce dernier (items peu détaillés) et afin de prouver qu'une analyse plus profonde des données était possible, nous avons utilisé ce questionnaire sur les données les plus récentes. L'analyse a été réalisée dans 13 établissements représentatifs du réseau, pour quatre segments de patientèle (Chirurgie, Médecine, Maternité et Ambulatoire), les attentes étant parfois différentes sur ces grands segments. Les questionnaires étaient remplis au moment de la sortie du patient. Seuls les résultats sur le segment chirurgie sont présentés ici, puisque l'accent est mis sur la méthode. Sur ce segment, durant l'année, 29102 questionnaires ont été récoltés par les cliniques. L'échantillon était composé de 50,6\% de femmes, l'âge moyen des patients était de 55 ans, 55,4\% d'entre eux étaient en chambre particulière. Le questionnaire était structuré autour de trois catégories d'information: la satisfaction globale, l'évaluation des différents éléments de la clinique (les items se trouvent dans la figure 3), les variables descriptives du profil des individus. Les niveaux de satisfaction étaient mesurés par des échelles de sourire en quatre points. La contribution des éléments à la satisfaction a été calculée grâce à une analyse factorielle des correspondances d'un tableau de contingence comprenant :

- en colonne, les modalités d'un indice global de satisfaction réduit à deux classes (satisfaction positive/satisfaction négative); 
- en ligne, les éléments susceptibles de jouer sur la satisfaction. Deux lignes ont été consacrées à chaque élément de l'expérience de service : l'une concerne son évaluation positive, l'autre son évaluation négative.

Le raisonnement suivant a été adopté : un seul axe factoriel suffit à expliquer 100\% de la variance de l'ensemble étudié et peut être interprété comme un axe de satisfaction. Sur cet axe de satisfaction figurent deux modalités de satisfaction globale (satisfaction positive et négative) ainsi que deux modalités de chaque élément (évaluation positive et négative). Plus les éléments de service sont positionnés aux extrémités de cet axe, plus ils contribuent positivement ou négativement à la satisfaction du client. Un pliage de l'axe permet la visualisation de chaque élément étudié, par un point sur une carte : l'abscisse d'un point correspond à la contribution de l'élément au niveau de satisfaction lorsqu'il est évalué de façon négative. Son ordonnée est sa contribution lorsqu'il est évalué de façon positive. Les coordonnées de l'indice global de satisfaction permettent d'établir les frontières. La figure 2 illustre le pliage de l'axe.

\section{Insérer Figure 2}

\section{RESULTATS}

Les résultats de l'étude quantitative montrent que les quatre logiques de contribution dont nous avons fait l'hypothèse (H1) sont présentes pour la satisfaction des patients des cliniques privés lors de séjour en chirurgie.

\section{Insérer Figure 3}

Examinons plus en détail la position des 19 critères de l'enquête.

Pour les patients de chirurgie, un seul élément est «Basique», la prise en compte de la douleur. Cet élément était déjà basique dans l'étude de Merdinger-Rumper [20]) qui comportait un nombre de critères plus élevé. Une mauvaise évaluation de ce critère entraine une forte insatisfaction, une bonne évaluation n'augmente pas fortement le niveau de satisfaction. Cependant, la frontière avec les éléments « clés » est proche. 
Parmi les éléments «Clés», nous retrouvons, pour l'essentiel, des critères liés à l'équipe médicosoignante et à son comportement (écoute, disponibilité, clarté des informations données) ainsi que l'attention accordée aux proches.

On trouve dans les éléments «Plus» un grand nombre de facteurs liés à l'activité hôtelière de l'hôpital, comme la restauration, le confort, la propreté et le calme de la chambre la journée et des critères liés à l'information et la communication (l'écoute particulière des médecins, les informations de sortie). Un seul élément hôtelier de confort (le calme dans le service de nuit) n'est pas dans les éléments «Plus» mais «Secondaire», juste à la frontière des deux catégories. Notre seconde hypothèse n'est donc que partiellement confirmée.

Les éléments «Secondaires» sont des critères essentiellement administratifs liés à l'accueil (téléphonique, central) et l'admission dans la clinique.

Le tableau I souligne les cinq critères qui jouent le plus sur l'insatisfaction du patient et ceux qui jouent le plus fortement sur sa satisfaction.

\section{Insérer Tableau I}

\section{DISCUSSION}

Quelques points particuliers sont remarquables :

Tout d'abord, on peut noter le caractère déterminant des qualités d'écoute, de disponibilité et de clarté des informations délivrées par le personnel médical. Cependant, des différences de niveau d'attentes du patient apparaissent selon la fonction. Ainsi une bonne écoute et la disponilité de l'équipe de jour, de nuit et des médecins sont chacun source de satisfaction forte, à des niveaux très proches. En revanche, une écoute et une disponibilité médiocre engendre une insatisfaction extrême s'il s'agit de l'équipe de jour, une plus grande tolérance, une exigence moindre, se manifeste pour l'équipe de nuit et enfin pour les médecins. La clarté des informations de l'équipe soignante engendre plus de satisfaction et d'insatisfaction que celle des médecins.

On peut ensuite souligner le caractère déterminant, «Clé », de l'attention réservée aux proches, ce critère méritant un investissement supplémentaire de la part des cliniques. Mieux informés, les proches peuvent être un relai précieux. 
Enfin parmi les éléments «Plus » la qualité des repas occupe une place extrême. C'est l'élément le plus dissymétrique, jouant fortement en positif, peu en négatif. Cela signifie que, dans une situation de non concurrence, l'investissement dans la restauration n'est pas une priorité à l'hôpital. Le client s'attend à un repas médiocre et n'est pas particulièrement mécontent si son expérience est mauvaise sur ce point. Si, par contre, le service chirurgie est dans une situation concurrentielle, souhaite faire la différence par rapport aux autres établissements et est jugé très satisfaisant sur les éléments «Clés », la restauration est un élément capable de générer une très forte satisfaction. En d'autres termes, on s'attend à mal manger mais un repas de qualité est une excellente surprise. Ainsi, on peut se demander si la pression sur les prix mise par l'hôpital privé sur ses sous-traitants est raisonnable si l'on souhaite vraiment un repas de qualité. C'est un arbitrage à réaliser.

Les éléments secondaires concernent des éléments administratifs mais aussi l'attention de l'équipe du bloc opératoire. On soulignera que ces éléments se passent au début de l'expérience du patient dans la clinique. La satisfaction globale du patient étant demandé à la fin de cette expérience, il existe un effet d'oubli, comme le montre des recherches récentes [24].

Il peut être signalé que d'un segment à l'autre, les cartes restent stables sur certains critères mais évoluent fortement sur d'autres critères. Ainsi, le confort de la chambre est encore plus influant sur la satisfaction d'une patiente de maternité qu'un patient de chirurgie (élément encore plus «Plus ») ou le calme dans le service de nuit passe de «Secondaire» à «Plus », validant, sur ce terrain là, notre hypothèse 2 .

\section{CONCLUSION}

Cet article a permis de mieux comprendre la construction de la satisfaction du patient de chirurgie. Nos résultats vérifient la coexistence d'éléments à contribution variable, dont le poids dans la satisfaction dépend de leur niveau de performance, et d'autres à contribution fixe.

Il permet aux directeurs d'établissements et aux responsables qualité d'y voir plus clair sur leur priorité d'actions. Ils devront d'abord chercher à maintenir un niveau acceptable et constant de performance sur les éléments «Basiques », c'est la moindre des choses pour le patient. Ces éléments doivent être totalement maîtrisés et verrouillés par l'entreprise de service. De bonnes performances 
sur les éléments «Clés » et «Plus » sont nécessaires pour engendrer la satisfaction du patient et peuvent constituer des critères de différenciation. Les éléments «Clés » sont à travailler en priorité ; ensuite, les «Plus » permettent de créer la surprise par leur bonne performance et poussent le niveau de satisfaction globale du patient vers le haut. Par ailleurs, investir pour améliorer la performance des éléments « Secondaires » est important mais non prioritaire.

A côté de l'attention et de l'information donnée au malade et à ses proches qui sont déterminants et prioritaires, l'étude souligne le rôle particulier des critères de confort hôtelier et notamment de la restauration comme élément de différentiation.

Les apports de cette recherche peuvent cependant être modérés par un certain nombre de limites, qui ouvrent des voies de recherches futures. Tout d'abord, soulignons le manque de validité convergente entre les méthodes à poids fluctuants, même si les méthodes les plus performantes (dont le modèle Tétraclasse) donnent des résultats proches [23]. La principale limite porte sur les pondérations des éléments : elles sont calculées à partir d'une synthèse des réponses de l'échantillon. Les différences entre patients sont donc éliminées. Or, ce qui est « Basique » pour un patient peut être «Clé » pour un autre. Il faut donc travailler par segment stratégique de patients et, au delà des quatre segments étudiés, il est probable que les cartes de contribution soient en partie différentes selon la durée de séjour, l'âge du patient, ou sa pathologie. Il serait également intéressant de comparer les critères de satisfaction du patient à ceux des accompagnants. Une autre limite porte sur le choix des critères de satisfaction. Etaient-ils exhaustifs, ne faudrait-il pas les détailler ? Un effort pourrait être réalisé dans ce domaine à l'avenir, le questionnaire peut être amélioré. Enfin, les attentes des patients évoluent (nouvelles technologies de l'information, normes de confort). Même si ces changements sont en général lents, et que l'écoute, l'attention et l'information données aux patients resteront toujours des éléments fondamentaux, il est souhaitable de répliquer cette étude à intervalles réguliers (entre 2 et 4 ans), en intégrant de nouveaux critères si nécessaire et en analysant l'évolution des priorités du patient en matière de satisfaction. 


\section{BIBLIOGRAPHIE}

1. Llosa S. L'analyse de la contribution des éléments de service à la satisfaction : un modèle Tetraclasse. Décisions Marketing 1997; $10: 81-88$.

2. Heskett J.L., Jones T.O., Loveman G.W., Sasser W.E. et Schlesinger L.A . Putting the service-profit chain to work. Havard Business Review 1994; 72, 2: 164-174.

3. Rust R.T., Zahorik A.J. et Keiningham T.L. Return on quality (ROQ) : making service quality financially accountable. Journal of Marketing 1995; 59, 2: 58-70.

4. Berard E., Gloanec M., Minvielle E. Usages des indicateurs de qualité en établissement de santé. Journal de l'Economie Médicale 2009 ; 1-2 : 5-20

5. Hunter J.E., The desperate need for replications. Journal of Consumer Research 2001; 28, 1 : 149-158.

6. Plichon V. Analyse de l'influence de la satisfaction des états affectifs sur le processus de satisfaction dans la grande distribution. Thèse de doctorat en sciences de gestion, Université de Bourgogne 1999

7. Ngobo P.V. Les standards de comparaison et la gestion de la satisfaction de la clientèle. Décisions Marketing 1998 ; 13 : 57-66.

8. Oliver R.L. et Swan J.E. Consumer perceptions of interpersonal equity and satisfaction in transactions : a field survey approach. Journal of Marketing 1989; 53, 2: 21-35.

9. Evrard Y. La satisfaction des consommateurs : état des recherches. Revue Française de Marketing 1993 ; 144-145, 4-5 : 53-65.

10. Howard J.A. et Sheth J.N.S. The theory of buyer behavior, New York, John Wiley 1969: 458

11. Oliver R.L. A cognitive model of the antecedents and consequences of satisfaction decisions. Journal of Marketing Research 1980;17, 4: 460-469.

12. Herzberg J., Mausner B. et Snyderman B. The motivation to works, New York, John Wiley and Sons. 1959: 160

13. Swan J.E. et Combs L.J., Product performance and consumer satisfaction : a new concept. Journal of Marketing 1976; 40, 2: 25-33.

14. Maddox R.N. Two factors theory and consumer satisfaction : replication and extension. Journal of Consumer Research 1981; 8, 1: 97-102.

15. Mittal V, Ross W, Baldasare P The asymmetric impact of negative and positive attribute-level performance on overall satisfaction and repurchase intention. Journal of Marketing 1998; 62: 33-47

16. Kano N., Seraku N., Takahashi F. et Tsuji S. Myrioku-teki Hinshitu to Atari-mae Hinshitu (Attractive quality and must-be quality), Hinshitu Quality, the Journal of the Japanese Society for Quality Control 1984; 14, 7, 39-48, version traduite dans : J.D. Hroni The best on quality, International Academy for Quality, 7, Milwaukee, WI : The Quality Press. 1996

17. Brandt R.D. How service marketers can identify value-enhancing service elements. The Journal of Services Marketing 1988;2, 3: 35-41.

18. Lichtlé M.C., Llosa S., Plichon V. La contribution des éléments d'une grande surface alimentaire à la satisfaction du client. Recherche et Applications en Marketing 2002; vol $17, n^{\circ} 4: 23-34$.

19. Léo, P.-Y, \& Philippe, J.. Positionnement concurrentiel des zones commerciales et satisfaction du consommateur. Recherche et applications en marketing $2003 ; 18,3: 45-63$.

20. Merdinger- Rumpler C. Contribution des éléments de service à la satisfaction du patient hospitalisé : une application du modèle tétraclasse. Décisions Marketing 2009 ; 53 : 43-52 
21. Bartikowski B., Llosa S. Customer Satisfaction Measurement: Comparing Four Methods of Attribute Categorizations Service Industries Journal 2004; vol 24, n 4: 67-82

22. Robinot, E., \& Giannelloni, J.-L. Do hotels" "green" attributes contribute to customer satisfaction? Journal of Services Marketing 2010; 24(2): 157-169.

23. Ray, D., \& Gotteland, D. Mesurer 1 'asymétrie des impacts des attributs sur la satisfaction: comparaison de la validité convergente de cinq méthodes. Recherche et applications en marketing $2005 ; 20: 1-20$.

24. Vo Thi T. Rôles de différents moments de l'expérience de service dans la satisfaction globale Thèse de doctorat, Aix Marseille Université, IAE d'Aix en Provence, 2012 
Tableau I. Les critères qui jouent le plus fortement sur la satisfaction et l'insatisfaction des patients hospitalisés en Chirurgie

\begin{tabular}{|l|l|}
\hline \multicolumn{1}{|c|}{$\begin{array}{c}\text { Critères influençant } \\
\text { l'INSATISFACTION }\end{array}$} & \multicolumn{1}{|c|}{$\begin{array}{c}\text { Critères influençant la } \\
\text { SATISFACTION }\end{array}$} \\
\hline $\begin{array}{l}\text { 1. Ecoute et disponibilité de } \\
\text { 1'équipe de jour }\end{array}$ & 1. Qualité des repas \\
\hline $\begin{array}{l}\text { 2. Clarté des informations de } \\
\text { 1'équipe soignante }\end{array}$ & 2. Confort de la chambre \\
\hline $\begin{array}{l}\text { 3. Ecoute et disponibilité de } \\
\text { 1'équipe de nuit }\end{array}$ & $\begin{array}{l}\text { 3. Clarté des informations de } \\
\text { 1'équipe soignante }\end{array}$ \\
\hline 4. Prise en compte de la douleur & $\begin{array}{l}\text { 4. Clarté des informations de } \\
\text { sortie }\end{array}$ \\
\hline $\begin{array}{l}\text { 5. Attention réservée aux } \\
\text { proches }\end{array}$ & $\begin{array}{l}\text { 5. Attention réservée aux } \\
\text { proches }\end{array}$ \\
\hline
\end{tabular}


Figure 1- Les différentes logiques de contribution à la satisfaction du modèle Tétraclasse (Llosa, 1997)

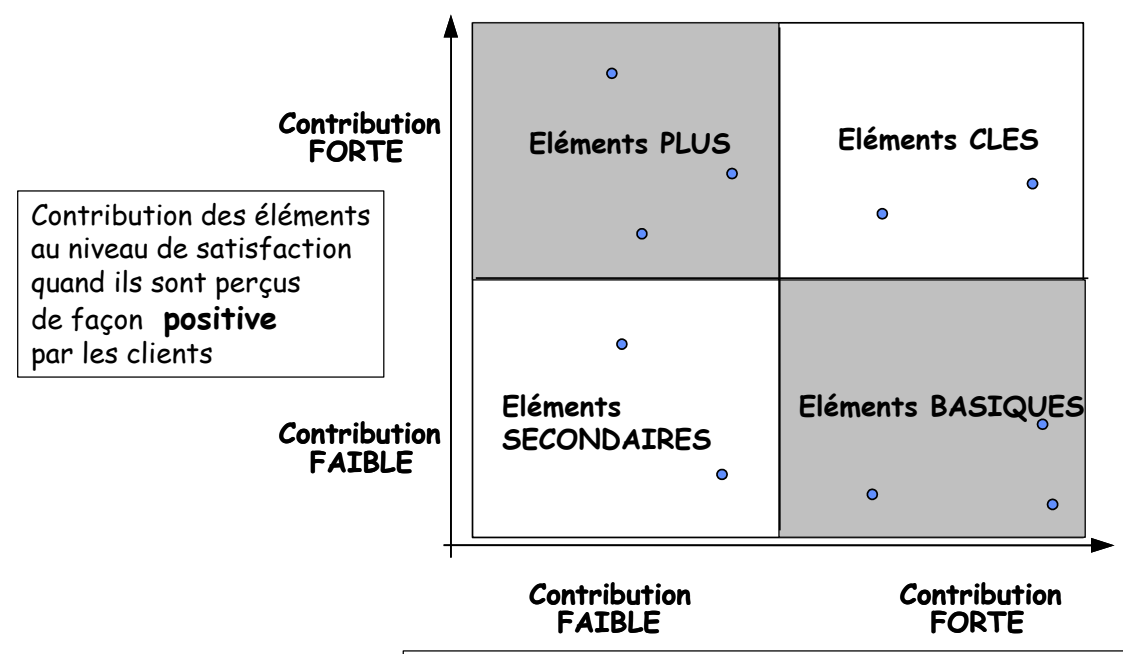

Contribution des éléments au niveau de satisfaction quand ils sont perçus de façon négative par les clients 
Figure 2. Représentation de l'axe factoriel de satisfaction puis de son pliage, avec contribution différente d'un élément selon la position de sa modalité négative et positive sur l'axe.

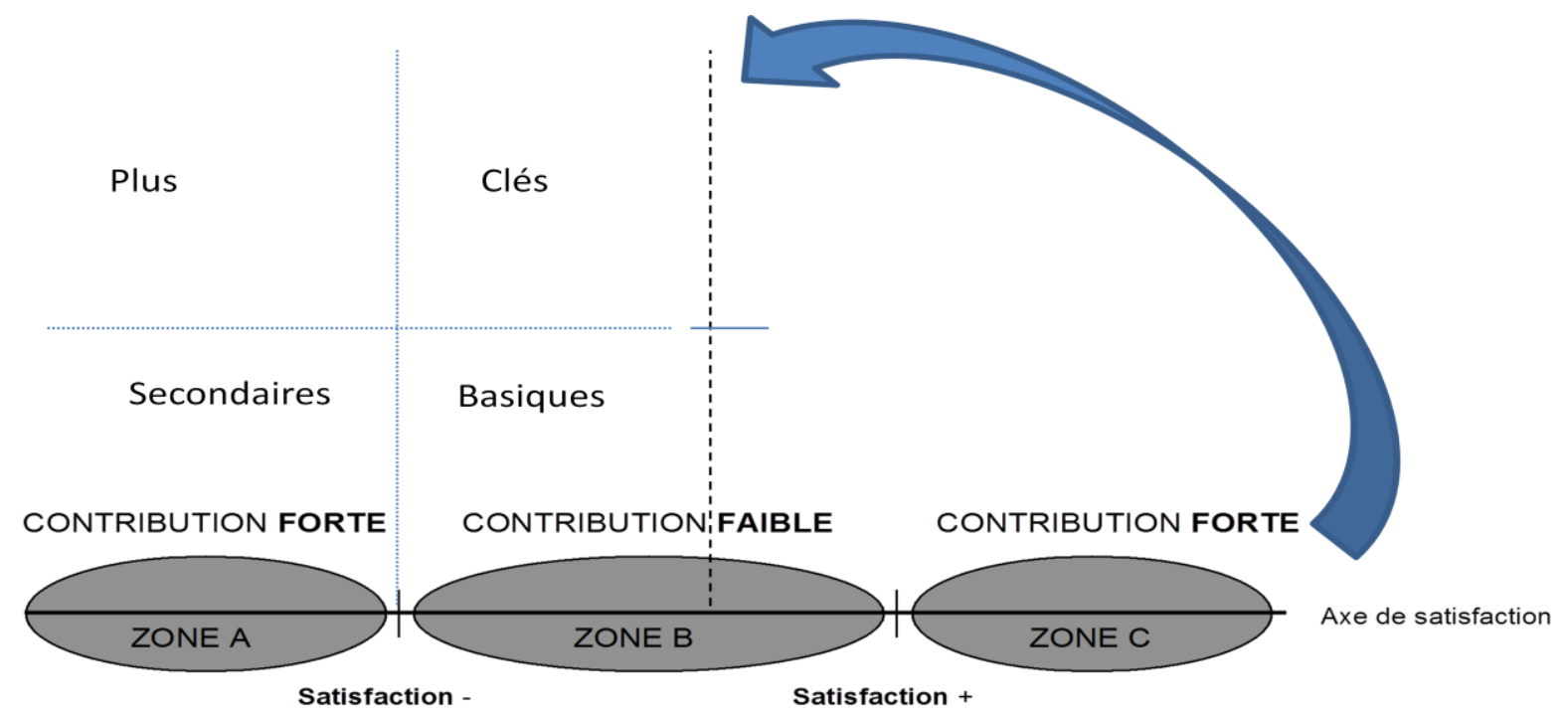


Figure 3. La contribution des éléments de service à la satisfaction des patients de Chirurgie

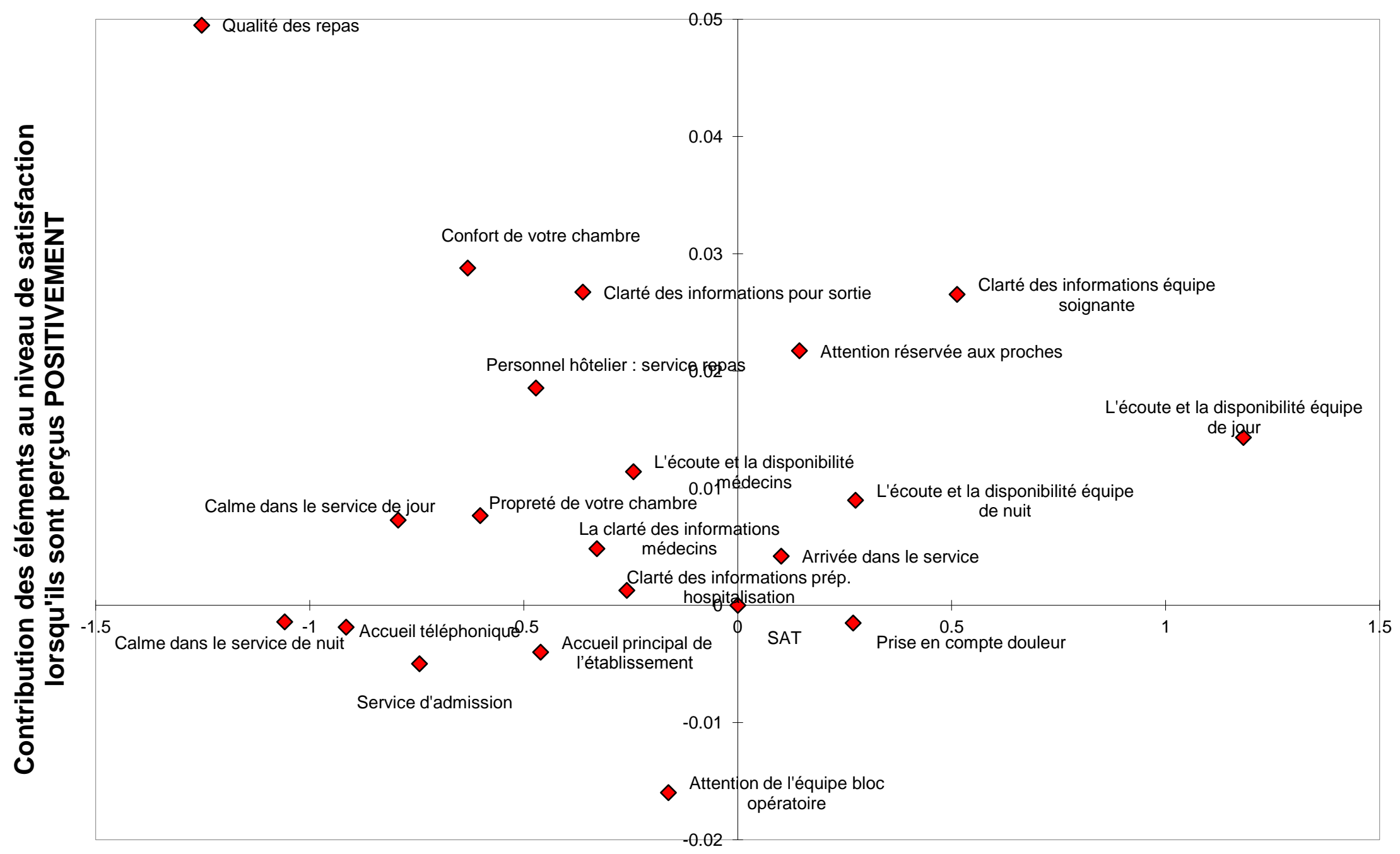

Contribution des éléments au niveau de satisfaction lorsqu'ils sont perçus NEGATIVEMENT 